\title{
Política, identidad y conflicto en el Japón del siglo XIX: Una propuesta de aprendizaje sobre modelos de modernización en el contexto de Asia Oriental
}

\section{Politics, Identity and Conflict in Japan throughout XIXth Century: A proposal for learning about modernization models in the context of East Asia}

CAROLINA GARCía SANZ

https://orcid.org/0000-0002-2378-6005

Universidad de Sevilla

Departamento de Historia Contemporánea

cgarciasanz@us.es

DOI: http://dx.doi.org/10.12795/9788447231003.028

Pp.: 593-613 


\section{Breve descripción del contexto del CIMA}

El Ciclo de Mejora en el Aula (CIMA) que se analiza en la presente comunicación se ha diseñado y llevado a cabo para la asignatura Historia Contemporánea de Asia Oriental del Grado de Estudios de Asia Oriental (Facultad de Filosofía), impartido en el Campus de Excelencia Internacional Andalucía Tech (Universidades de Sevilla y Málaga). Es una asignatura obligatoria de tercer curso. El Grado se orienta a la formación pluridisciplinar y a la capacitación de profesionales en distintas facetas que comprenden las relaciones internacionales con China, Japón y Corea (político-legal, financiera, comercial, científica, cultural, etc.). El programa de la asignatura plantea, por tanto, una aproximación a la historia contemporánea de los tres países de referencia que permita la interpretación y análisis de los acontecimientos a un nivel profundo y holístico, aportando a los alumnos un sentido crítico y ético de la historia y la cultura de las sociedades, con las que van a entablar relaciones e interactuar durante su ejercicio profesional. El enfoque en la propuesta docente en un Grado de implantación reciente ha de entenderse, por tanto, desde los retos actuales del mundo globalizado en el que vivimos y atendiendo a las exigencias del marco de enseñanza superior europeo (Grupo de alto nivel para la modernización de la enseñanza superior de la UE, 2013). Además, la asignatura brinda una formación inicial a potenciales historiadores del área de conocimiento (si bien, éste representaría el perfil más minoritario entre el alumnado).

Durante el ciclo de mejora de ocho horas cuya experiencia se refleja en esta contribución, se ha trabajado sobre el aprendizaje de contenidos conceptuales, procedimientos intelectuales y actitudes y valores relativos al proceso de modernización de Japón en el siglo XIX, coincidiendo con la apertura al exterior de la Era Meiji. Los contenidos trataban de aproximar al alumnado a los siguientes aspectos: 1. Identidad y alteridad cultural tras el fin de la política de aislamiento de los Tokugawa. Impacto

Ciclos de Mejora en el Aula (2020). Experiencias de Innovación Docente de la US Esta obra se distribuye con la licencia Creative Commons 
de la intervención extranjera y respuestas locales; 2 . Reforma política y transformación económica: fórmulas de integración regional en el sistema de relaciones internacionales; 3. Nuevos modelos sociales e intelectuales: convergencias y divergencias regionales. Uno de los aspectos claves para el planteamiento de la intervención fue la selección y organización de los contenidos, con el propósito de fomentar un aprendizaje interactivo y colaborativo en el aula virtual (impuesta por la situación epidemiológica actual) siguiendo los principios didácticos recogidos por las propuestas de Bain (2005, pp.113-132). Del mismo modo, la elección de la metodología del trabajo por problemas en grupos de alumnos (Finkel, 2008, pp.153-170) debían estar claramente vinculadas con los objetivos a alcanzar en la asignatura (García Martínez, 2007).

\section{Diseño previo del CIMA}

El modelo metodológico elegido y posible se ha basado en la reelaboración de las ideas previas de los estudiantes a partir del planteamiento de un problema general (PG) que se subdivide, a su vez, en dos (P1; P2) para facilitar su aprendizaje autónomo. El alumno debía aproximarse de una forma crítica al proceso de transformación política, económica, social y cultural emprendido por Japón a partir de la presión de la potencia estadounidense desde 1853. La propuesta didáctica incidía en la compresión de los factores de la crisis interna de un sistema político de clanes, del conflicto interno sobre las ideas de tradición y el cambio precipitado por la firma de tratados desiguales, del choque cultural en la adaptación a un régimen oligárquico de inspiración occidental, de la filosofía detrás del sincretismo en la adopción de la ciencia y la técnica extranjera y a la vez el mantenimiento de las esencias o de las dinámicas de generación -cuando no imposición- de un consenso social mediante la conexión entre un proyecto militarista y expansivo y la identidad nacional. El alumno, por tanto, debe no sólo conocer las dinámicas 
de transformación sino también ser capaz de razonar críticamente sobre los efectos del mismo -más allá del papel jugado por las élites- en el conjunto de las sociedades japonesa, china y coreana en la transición entre los siglos XIX y XX. Como ya se ha señalado, el planteamiento de la intervención parte de una concepción constructivista del conocimiento y del aprendizaje basado en la investigación (Porlán, 1993).

Los problemas formulados al estudiante fueron los siguientes:

PG: Japón suele considerarse un modelo de modernización en el contexto de Asia Oriental en la segunda mitad del siglo XIX „ ¿qué consideras que puede haber de positivo $y$ de negativo de esta experiencia para las sociedades contemporáneas? ¿Por qué?

P1: Imagínate que eres uno de los elegidos por el Shogun para negociar el llamado Tratado Harris de 1858, ¿qué estrategia de negociación habrías seguido? ¿Qué aspectos del Tratado que finalmente se suscribió te hubieran resultado más difícil de aceptar y por qué? ¿Qué tipo de política apoyarías en el futuro para tratar de revocar aquellos aspectos que te parecieron más inaceptables de ese acuerdo?

P2: El Príncipe Ito Hirobumi en 1885 te encarga la redacción de un discurso en el que se trata de convencer a la opinión pública urbana de las bondades de la futura constitución de 1889, ¿cuáles son los aspectos respecto al modelo político-constitucional japonés que te parecerían más controvertidos y dificiles de aceptar por quienes no integran la élite gobernante? ¿Por qué?

Los estudiantes debían hallarse en un estado de "activación mental" (Alba y Porlán, 2017, pp.40-41) ante un PG que trataba de despertar su interés. Las hipótesis previas que formularon tenían que ser contrastadas con la nueva información obtenida conforme trabajaban para resolver estos problemas o preguntas (PG; P1; P2). El estudiante se convierte en "sujeto epistémico" (Porlán, 2017, p.73), que construye su propio conocimiento. Para que ese principio se cumpla, el docente ha de realizar previamente una reflexión profunda y rigurosa sobre los contenidos (datos, conceptos, procedimientos intelectuales y valores), las

Ciclos de Mejora en el Aula (2020). Experiencias de Innovación Docente de la US Esta obra se distribuye con la licencia Creative Commons 


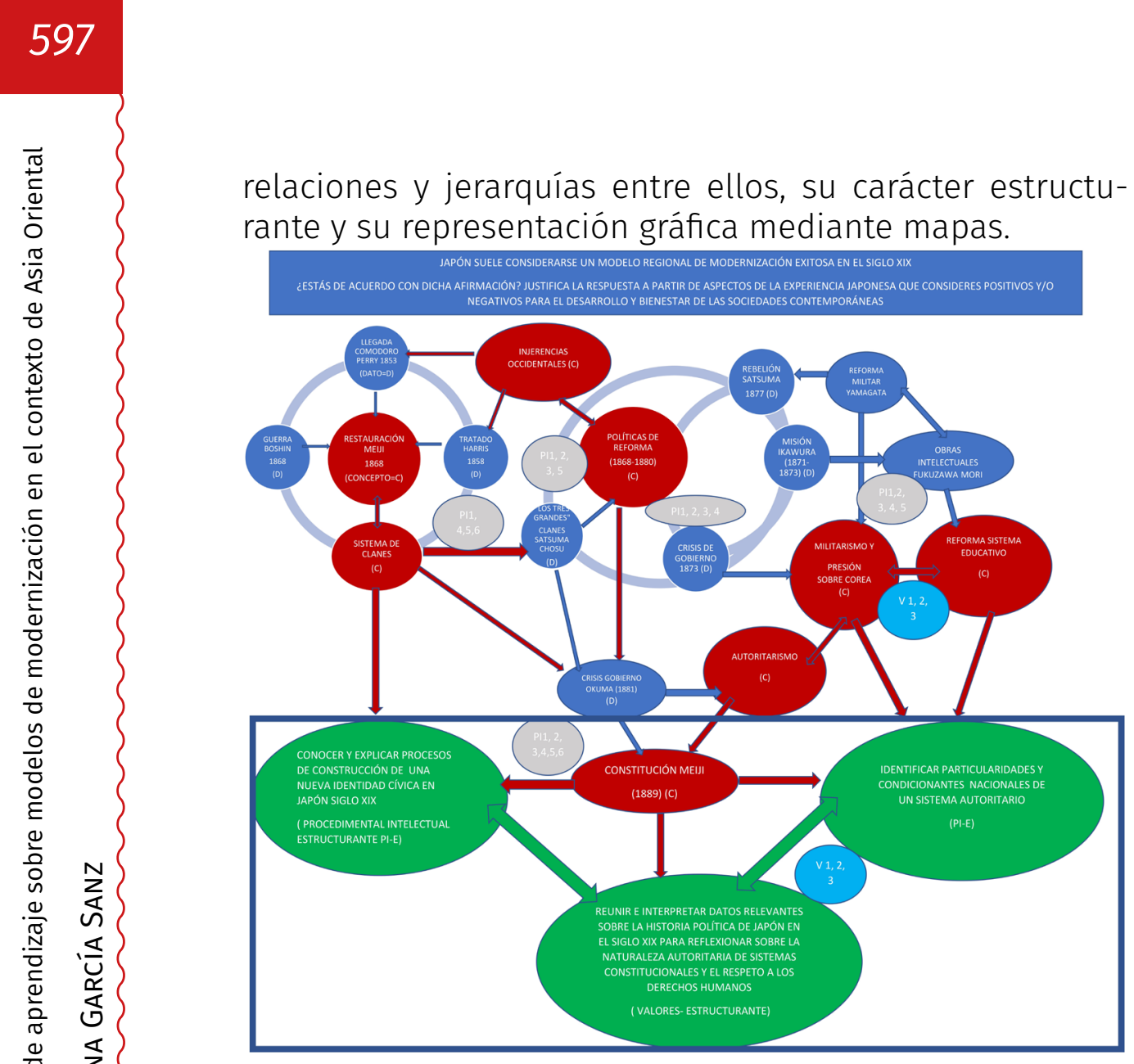

Figura 1. Mapa de Contenidos

Datos

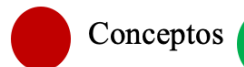

Procedimientos Intelectuales

Valores

Procedimientos intelectuales:

- Análisis crítico de documentos históricos PI 1

- Descripción y análisis de recursos audiovisuales y en particular de la fotografía con valor histórico PI 2

- Contextualizar y analizar históricamente fuentes artísticas y literarias P3

- Localización, discriminación y análisis de información a partir de motores de búsqueda y recursos Ciclos de Mejora en el Aula (2020). Experiencias de Innovación Docente de la US
Esta obra se distribuye con la licencia Creative Commons 
académicos cuyo acceso exige el empleo de las nuevas tecnologías de información PI 4

- Desarrollo de la capacidad expositiva (expresión oral y escrita en castellano e inglés) PI 5

- Reflexión sobre de la diversidad del enfoque histórico según los contextos socioculturales PI6

- Valores éticos:

- Tolerancia y respeto hacia las formas socioculturales distintas a la propia VE 1

- Actitud receptiva y crítica hacia la información procedente de otros contextos culturales

- VE 2

- Respeto a los Derechos Humanos y a los principios de accesibilidad universal, igualdad, no discriminación y los valores democráticos y de la cultura de la paz VE 3

Del modelo metodológico elegido y posible se deriva el diseño y realización de actividades de contraste (AC) que desafien los esquemas de pensamiento e ideas iniciales (IA) de los alumnos, incluyendo una fase de trabajo individual (TI) y otra colaborativa de discusión grupal (DG). Además, las dinámicas de debate colectivo se orientarían a facilitar la elaboración de conclusiones con las ideas finales. Esta fase previarequirió, además, un elevado nivel de concreción a la hora de plantear los problemas, de modo que al alumnado le resultara más fácil conectarlos con los contenidos históricos trabajados. También fue necesario atender a la formulación de los enunciados de las AC, para orientar con claridad al estudiante durante el manejo de diferentes fuentes de información. Durante el planteamiento del PG, P1 y P2 introduje explicaciones de claves teóricas (E-P1/E-P2) para facilitar la organización del proceso de documentación, así como la interpretación de los datos más relevantes.

Ciclos de Mejora en el Aula (2020). Experiencias de Innovación Docente de la US Esta obra se distribuye con la licencia Creative Commons 


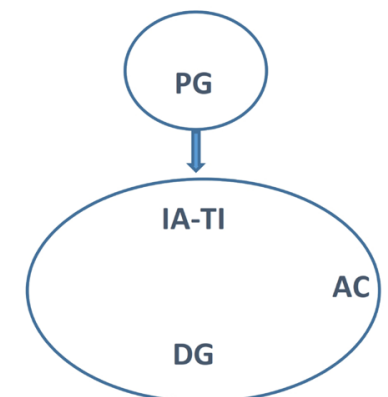

Este mismo ciclo se repite con el planteamiento del sub-

problema E-P2

\section{SIGLAS DEL DIAGRAMA EMPLEADO PARA EL DISEÑO DEL MODELO}

PG: Presentación y planteamiento del Problema General

AC: Actividades de Contraste (Trabaja Individual sabre fuentes históricas; DG:

Discusión Grupal)

E-P1: Explicación Planteamiento Sub-Problema 1

AC: Actividades de Contraste (Trabajo Individual sabre fuentes históricas; DG:

Discusión Grupal)

E-P2: Explicación Planteamiento Sub-Problema 2

AC: Actividades de Contraste (Trabaja Individual sabre fuentes históricas; DG:

Discusión Grupal)

C: Conclusiones

Figura 2. Modelo Metodológico Posible

La secuencia de actividades propuestas estaba vinculada a los problemas planteados y se programaron en correspondencia con las distintas fases del modelo metodológico seleccionado.

Ciclos de Mejora en el Aula (2020). Experiencias de Innovación Docente de la US (c) (i) $\Theta$ Esta obra se distribuye con la licencia Creative Commons 


\section{SESIÓN 1}

\begin{tabular}{|l|l|c|c|}
\hline 1 & PRESENTACIÓN DEL PROBLEMA GENERAL & PG & 20' \\
\hline Tras haberles pasado el cuestionario inicial (CI) de ideas previas en la clase \\
anterior, se introduce la problemática general a través de algunas de las \\
respuestas que los alumnos han escrito anónimamente en el Cl. Para ello, \\
se proyectan en la pantalla una selección de respuestas, unas erróneas y \\
otras acertadas. \\
\hline $\begin{array}{l}\text { Recursos: Cuestionarios de Ideas Previas respondidos en la clase anterior, } \\
\text { Diapositiva Powerpoint, Plataforma virtual }\end{array}$ \\
\hline
\end{tabular}

\begin{tabular}{|c|c|c|c|}
\hline 2 & $\begin{array}{l}\text { ACTIVIDAD SOBRE LAS IDEAS PREVIAS } \\
\text { INDIVIDUALES-DG }\end{array}$ & $A C$ & $90^{\prime}$ \\
\hline \multicolumn{4}{|c|}{$\begin{array}{l}\text { Los alumnos previamente divididos en grupos de cuatro deberán seleccionar } \\
\text { una respuesta que creen acertada y otra errónea. Deben discutir entre } \\
\text { ellos y ponerse de acuerdo sobre su elección y argumentarla. Para ello, } \\
\text { los componentes del grupo trabajando en pareja deben localizar en una } \\
\text { página web de referencia un documento de carácter histórico que apoye } \\
\text { la argumentación conjunta respecto a las dos respuestas seleccionadas. Al } \\
\text { final de este proceso, que durará una hora, deben redactar la actividad y } \\
\text { enviarla por correo electrónico. } \\
\text { Durante la siguiente media hora se desarrollará un debate con un portavoz } \\
\text { de cada grupo, cuyo orden de intervención vendrá dado por el lugar que } \\
\text { ocupan las respuestas elegidas en la diapositiva del Powerpoint. La puesta } \\
\text { en común servirá para crear un archivo de clase sobre los documentos } \\
\text { históricos de Japón en el siglo XIX. }\end{array}$} \\
\hline \multicolumn{4}{|c|}{$\begin{array}{l}\text { Recursos: Plataforma EV de la universidad, powerpoint, http://www.mext. } \\
\text { go.jp/ } \\
\text { Modern Japan in Archives https://www.ndl.go.jp/modern/e/index.html }\end{array}$} \\
\hline
\end{tabular}

\section{SESIÓN 2 -P1}

\begin{tabular}{|l|c|c|c|}
\hline 3 & \multicolumn{1}{|c|}{ PRESENTACIÓN DEL PROBLEMA 1 } & E-P1 & 20' \\
\hline $\begin{array}{l}\text { Enlazando con las conclusiones obtenidas en la AC del día anterior, se } \\
\text { les planteará el problema de las respuestas locales ante las injerencias } \\
\text { estadounidenses. Se les presentará y explicará el documento histórico } \\
\text { del Tratado Harris y datos de contexto de este primer sub-problema, que } \\
\text { deberán trabajar en los mismos grupos de la sesión anterior. }\end{array}$ \\
\hline $\begin{array}{l}\text { Recursos: Plataforma de EV, powerpoint, https://archive.org/details/ } \\
\text { completejournaloharr }\end{array}$ \\
\hline
\end{tabular}

Ciclos de Mejora en el Aula (2020). Experiencias de Innovación Docente de la US Esta obra se distribuye con la licencia Creative Commons 


\begin{tabular}{|c|c|c|c|}
\hline 4 & & $-C$ & $0^{\prime}$ \\
\hline \multicolumn{4}{|c|}{$\begin{array}{l}\text { Los alumnos tendrán que preparar un informe de la situación que explica el } \\
\text { Tratado Harris, para ello se les remitirá a los archivos diplomáticos modernos } \\
\text { japoneses y deberán establecer por parejas dos escenarios contrafactuales } \\
\text { internacionales teniendo en cuenta los factores políticos internos. Además, } \\
\text { en el diseño de esos escenarios con sus propias propuestas de cambio } \\
\text { político, deben de repartirse los papeles de cuatro figuras históricas } \\
\text { destacadas en el contexto del P1, y cuyo listado se les ha entregado por } \\
\text { escrito con las instrucciones de la actividad. } \\
\text { Tras } 60 \text { minutos de trabajo individual y por grupos, se crearán cuatro grandes } \\
\text { subgrupos en función de cada una de las figuras históricas asignadas. Los } \\
\text { alumnos que representan cada papel deberán discutir sus propuestas de } \\
\text { cambio político para el fortalecimiento. La segunda parte de la actividad } \\
\text { tendrá una duración aproximada de } 40 \text { minutos ( } 25 \text { minutos para la discusión } \\
\text { en subgrupos y } 15 \text { minutos para la puesta en común de las conclusiones) }\end{array}$} \\
\hline \multicolumn{4}{|c|}{$\begin{array}{l}\text { Recursos: Plataforma de enseñanza virtual, instrucciones de la actividad, } \\
\text { fotografias de la Galería on-line de la Dieta japonesa sobre las grandes } \\
\text { figuras del Japón moderno Portraits of Modern Japanese Historical Figures } \\
\text { https://www.ndl.go.jp/portrait/e/ }\end{array}$} \\
\hline
\end{tabular}

SESIÓN 3- P2

\begin{tabular}{|c|c|c|c|}
\hline 5 & Presentación del Problema 2 & $\mathrm{E}-\mathrm{P} 2$ & $10^{\prime}$ \\
\hline \multicolumn{4}{|c|}{$\begin{array}{l}\text { Enlazando con las conclusiones obtenidas en la AC del día anterior, se } \\
\text { les introducirá una película coreana de } 1959 \text { (Gojonghwangje-wa uisa An } \\
\text { Junggeun) sobre el legado de la figura de Ito Hirobumi y la sociedad japonesa } \\
\text { desde la perspectiva coreana para trabajar algunos de los aspectos más } \\
\text { controvertidos del imperialismo japonés. }\end{array}$} \\
\hline \multicolumn{4}{|c|}{ Recursos: plataforma de Enseñanza Virtual y powerpoint } \\
\hline 6 & $\begin{array}{c}\text { Visionado de la película King Gojong and martyr An } \\
\text { Jung-Geun (Gojonghwangje-wa uisa An Junggeun) } \\
\text { (1959) }\end{array}$ & $A C$ & $110^{\prime}$ \\
\hline \multicolumn{4}{|c|}{$\begin{array}{l}\text { Visionado del filme, si bien previamente se les aportará a los alumnos } \\
\text { un guion de elementos a los que deben prestar atención relacionados } \\
\text { con el sistema político japonés, el papel de la élite política y militar, las } \\
\text { relaciones entre poder civil y ejército, y las consecuencias en el plano social } \\
\text { y humanitario de la ideología nacionalista durante la época Meiji. } \\
\text { Se les pide que tomen anotaciones que deberán utilizar para el trabajo en } \\
\text { la última sesión. }\end{array}$} \\
\hline
\end{tabular}

Ciclos de Mejora en el Aula (2020). Experiencias de Innovación Docente de la US Esta obra se distribuye con la licencia Creative Commons Reconocimiento-NoComercial-SinObraDerivada Internacional (CC BY-NC-ND 4.0.) 
Recursos: enlace desde la plataforma EV a la película King Gojong and martyr An Jung-Geun (Gojonghwangje-wa uisa An Junggeun) (1959)

https://www.koreanfilm.or.kr/eng/films/index/filmsView. jsp? movieCd=20147710

https://www.youtube.com/watch?v=vuLZXGr46rU

SESIÓN 4-P2

\begin{tabular}{|c|c|c|c|}
\hline 7 & $\begin{array}{l}\text { Discusión Grupal sobre las represer } \\
\text { reanas en la película King Gojong an } \\
\text { ang-Geun (Gojonghwangje-wa uisa Ar } \\
\text { (1959) de la dominación japon }\end{array}$ & $A C$ & $30^{\prime}$ \\
\hline \multicolumn{4}{|c|}{$\begin{array}{l}\text { La idea es establecer un debate grupal sobre la naturaleza del colonialismo } \\
\text { en el contexto de Asia Oriental y sus traumáticos efectos socio-psicológicos } \\
\text { en la región. Se plantea una discusión que aborda cuestiones tan intrincadas } \\
\text { como la representación del asesino de Ito Hirobumi como un mártir y un } \\
\text { patriota en las dos Coreas. Se les mostrarán algunos clips de youtube en los } \\
\text { que se banaliza sobre el asesinato político, incluso con actores actuales que } \\
\text { han interpretado ese papel en series históricas de Corea del Sur con gran } \\
\text { éxito internacional a través de plataformas como Netflix. }\end{array}$} \\
\hline \multicolumn{4}{|c|}{$\begin{array}{l}\text { Plataforma EV, Ito Hiro } \\
\text { uksoo's \#WhoAreYou \#Kim } \\
\text { nj_iMtVoCFo }\end{array}$} \\
\hline
\end{tabular}

8

Trabajo individual sobre el P2

$A C$

$60^{\prime}$

El Príncipe Ito Hirobumi en 1885 te encarga la redacción de un discurso en el que se trata de convencer a la opinión pública urbana de las bondades de la futura constitución de 1889. ¿Cuáles son los aspectos respecto al modelo político-constitucional japonés que te parecerian más controvertidos y difíciles de aceptar por quienes no forman parte de la élite dominante? ¿Por qué?

El alumno dispondrá de 90 minutos para responder al problema planteado, para ellos dispondrá de acceso al texto constitucional y además deberá razonar empleando el conocimiento adquirido a través de las distintas actividades realizadas. Se proporcionan una serie de fragmentos literarios extraídos de los literatos Natsume Soseki y Yosano Akiko (en este último caso introduciendo la perspectiva de género). El alumno deberá enviar su respuesta a la profesora.

Recursos: plataforma EV, https://www.ndl.go.jp/constitution/e/etc/c02. html, la reproducción de los textos de la obra de los dos autores señalados.

Ciclos de Mejora en el Aula (2020). Experiencias de Innovación Docente de la US Esta obra se distribuye con la licencia Creative Commons Reconocimiento-NoComercial-SinObraDerivada Internacional (CC BY-NC-ND 4.0.) 
El trabajo por problemas, como el propuesto en estas sesiones, a través del diseño de "experiencias que enseñan" (Finkel, 2008), requiere por parte del docente una aproximación a las ideas y esquemas mentales del alumnado para, desde sus realidades más próximas, en el sentido propuesto por la teoría constructivista del andamiaje y del concepto de zona de desarrollo próximo (ZDP) de Vigotsky, anclar conocimientos profundos e indelebles. Se parte de sus hipótesis iniciales para, desde ellas, ayudarles a construir un aprendizaje duradero y útil conectado con sus experiencias personales (no solamente operante en el ámbito académico). Un diagnóstico inicial de esas ideas resulta determinante para el diseño de la sesión y el desarrollo de las actividades de acuerdo con el modelo metodológico. Con ese propósito, se elaboró un cuestionario compuesto por ocho preguntas conectadas directamente con los problemas que se plantearía a los alumnos durante el CIMA. Los alumnos dispondrían de treinta minutos durante la clase anterior al inicio de la experiencia para responder el cuestionario y enviarlo de forma anónima. El mismo cuestionario sería contestado al final del ciclo. Era muy importante que no se sirvieran de ningún tipo de recurso externo para preservar los objetivos de la actividad. A continuación, se muestra el cuestionario.

1. Netflix se pone en contacto contigo, en calidad de especialista en Asia Oriental, para que asesores a una producción europea sobre el final de la política de "Sakoku" o reclusión japonesa en el siglo XIX ¿Cuáles serían los aspectos claves para el desarrollo del guion? Debes indicar y explicar las cuestiones más importantes para la ambientación histórica.

2. Imaginate que eres un samurái de un clan periférico en 1868 ¿qué bando hubieras escogido en la Guerra "Boshin" entre los partidarios del Shogunato y quienes pretendian la restauración imperial? ¿Por qué?

3. Ponte en el lugar de ese mismo sámurai ¿cómo te sentirías y qué posición habrías adoptado respecto 
al cambio de uno de tus iguales y reflejado en las fotografías tras lograr el poder?

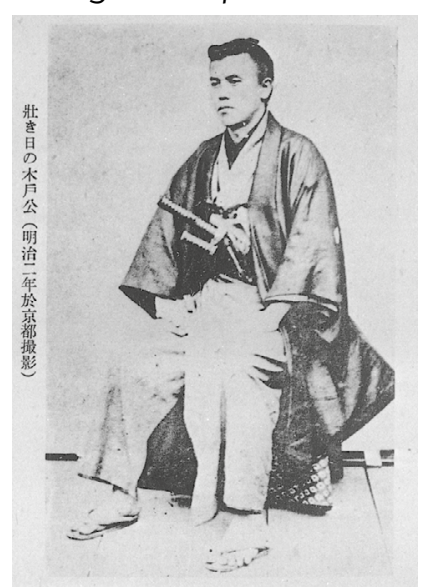

Figura 3. Kido Takayoshi

https://www.ndl.go.jp/portrait/e/datas/65.html?c=3

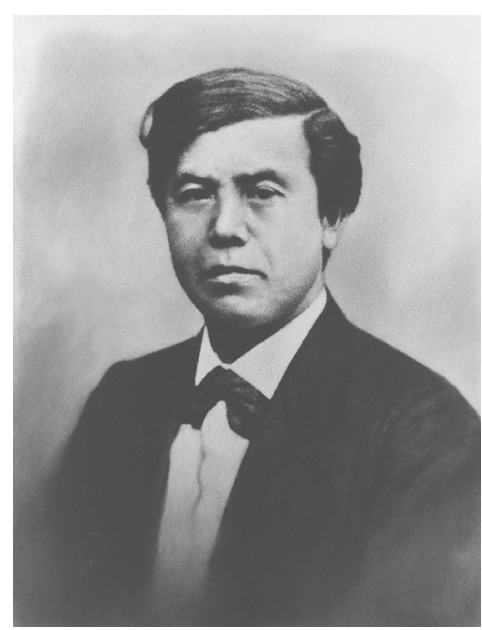

Figura 4. Kido Takayoshi

https://www.ndl.go.jp/portrait/e/datas/65.html?c=3

4. Tras haber colaborado con la Restauración Meiji, el gobierno te elige para llevar a cabo un estudio económico con objeto de facilitar la puesta en marcha del nuevo sistema financiero. ¿De qué país te interesaría estudiar su sistema bancario para su implantación en Japón? ¿Por qué?

Ciclos de Mejora en el Aula (2020). Experiencias de Innovación Docente de la US Esta obra se distribuye con la licencia Creative Commons 
5. Cuando asume la jefatura del gobierno en 1885, el Príncipe Ito Hirobumi te hace llamar para que integres un grupo de trabajo diplomático encargado de las relaciones con Corea. Dentro de ese equipo tu misión consistirá en redactar un discurso sobre el interés para la nación de la expansión sobre Corea. ¿Qué argumentos introducirías en el discurso? ¿Por qué? ¿Variarías los argumentos si el discurso en vez de ser leído ante la Dieta japonesa lo fuera ante un gobierno extranjero durante un viaje de Estado a Europa? ¿Cuáles emplearías en el segundo de los supuestos?

6. Teniendo en cuenta el buen resultado de tu labor propagandística a nivel nacional en relación a la cuestión coreana, Ito Hirobumi te encarga que hagas un estudio sobre las herramientas en manos del gobierno para convencer al conjunto social de que su política y el régimen constitucional, que pronto se aprobará, es el más idóneo para Japón. ¿Qué tipo de herramientas públicas o recursos del Estado le aconsejarías usar con ese propósito? ¿Por qué?

7. Imaginate que eres un/a preso/a político/a encarcelado/a como consecuencia de las leyes de orden público, que restringieron el derecho de asociación y reunión en Japón entre 1880 y 1887. Uno de tus compañeros/as de prisión está a punto de salir y ha decidido marcharse al exilio en Inglaterra. Escribe una nota para un periódico inglés explicando/denunciando las razones que te habrían llevado a esa situación.

8. Japón suele considerarse un modelo de modernización en el contexto de Asia Oriental en la segunda mitad del siglo XIX ¿qué consideras que puede haber de positivo y de negativo de esta experiencia para las sociedades contemporáneas? ¿Por qué? 


\section{Aplicación del CIMA}

La información de este apartado plasma los diarios de las sesiones en los que debía describir y analizar por escrito la práctica docente (Porlán y Martín, 1991). La asignatura tiene un total de 69 alumnos matriculados, de los cuales sólo 40 asisten con regularidad a clase y, de ellos, 32 decidieron participar en las actividades propuestas en este ciclo. De acuerdo con la planificación del CIMA, debía comenzar introduciendo al alumnado en la problemática general sobre si Japón podía considerarse un modelo de modernización en el contexto de Asia Oriental en el siglo XIX, a partir de los aspectos positivos y negativos de esta experiencia para las sociedades contemporáneas. En la primera sesión les proyectaría la primera actividad a partir de lo que habían respondido en el Cl. Los alumnos previamente divididos en grupos de cuatro debían seleccionar una respuesta que creían acertada y otra errónea. Debían discutir entre ellos y ponerse de acuerdo sobre la elección del equipo, así como explicar las razones de esta. Para ello, los componentes del grupo trabajaban en pareja teniendo que localizar un documento de carácter histórico. A los alumnos le llamó mucho la atención la creación de los grupos en la plataforma virtual, y lo cierto es que, tras el desconcierto inicial, la actividad funcionó muy satisfactoriamente. Las respuestas demostraron un elevado nivel en la argumentación y razonamiento histórico. Los documentos que fuimos incorporando al archivo eran los distintos tratados internacionales firmados por Japón desde el Tratado de Kanagawa (1854), fotografías de las diferentes misiones enviadas al exterior (en particular la Misión Iwakura), publicaciones sobre la crisis de gobierno de 1873, la reforma del sistema educativo y del ejército, o del debate previo a la promulgación de la constitución de 1889.

En la segunda sesión se realizó la puesta en común de los cuatro grupos. Comenzábamos con un desfase de 30 minutos respecto a la programación prevista en el diseño del CIMA. Enlazando con las conclusiones obtenidas sobre

Ciclos de Mejora en el Aula (2020). Experiencias de Innovación Docente de la US Esta obra se distribuye con la licencia Creative Commons 
los detonantes del proceso de modernización japonés, se planteó el problema de las respuestas locales ante las injerencias estadounidenses. El tiempo inicialmente previsto para mi contextualización fue inferior al que se precisaría durante la experiencia. Los alumnos tenían que imaginarse que habían sido elegidos por el Shogun para negociar el Tratado Harris con Estados Unidos en 1858. Presenté y expliqué el contexto del documento histórico. A continuación, ellos tenían que preparar un informe de la situación y los dilemas de la negociación de este. Para ello, trabajarían sobre la página web de la Dieta japonesa y de los archivos diplomáticos modernos japoneses. Debían establecer por parejas dos escenarios contrafactuales internacionales atendiendo a los factores políticos internos. Además, en el diseño de esos escenarios se asignaron respectivamente cuatro figuras históricas: (1) Yoshinobu Tokugawa, (2) Sakamoto Ryoma; (3) SaigoTakamori; (4) KidoTakayoshi. La actividad tenía prevista una duración total de 90 minutos, cuando sólo disponíamos ese día de 60. De ahí, que sólo hiciéramos la primera parte de la actividad. La dinámica de trabajo en grupo la percibí como satisfactoria y el nivel de logro en el análisis también fue elevado.

En la tercera sesión, los resultados en cuanto al nivel de logro reflejado en los documentos que me enviaron a partir del trabajo por figuras históricas no fueron tan satisfactorios (salvo en el caso de uno de los cuatro grupos). Esta parte de la actividad les obligaba más a trabajar individualmente en la preparación de sus personajes y propuestas. Continuando con la dinámica de enlazar el siguiente problema con las conclusiones que los alumnos extraían, planteamos la cuestión sobre cuáles eran los aspectos del modelo político-constitucional japonés que les parecerían más controvertidos y difíciles de aceptar, sobre todo para los sectores excluidos y marginados por un sistema de élites. La actividad inicialmente prevista consistía en el visionado íntegro de una película clásica coreana de 1959 sobre el período en el que Ito Hirobumi fue Residente General de Japón en Corea. Teniendo en cuenta 
el tiempo invertido en finalizar la actividad anterior y en la explicación de la siguiente, disponíamos aproximadamente de cuarenta minutos. De ahí que optara por el visionado de fragmentos significativos (que había seleccionado el día anterior, atendiendo al problema de tiempo que me venía atenazando durante toda la aplicación del CIMA). Se indicó a los alumnos los aspectos a los que debían prestar atención para utilizarlos en la actividad en grupo: (1) Discurso japonés sobre el tipo de relación que debe establecerse entre el Imperio japonés y Corea; (2) Narrativa coreana sobre lo que encarna el Tratado de Eulsa (1905) para su pérdida de soberanía (Japón asumiría la representación internacional de Corea y se suprimirían las fuerzas armadas coreanas) ; (3) Valoración del lenguaje cinematográfico empleado para transmitir un mensaje político interpelando directamente a las emociones (caracterización de los personajes, escenografía...etc.).

En la cuarta y última sesión se retomó la dinámica de trabajo grupal estableciendo posteriormente un debate acerca de la naturaleza del colonialismo en el contexto de Asia Oriental y sus profundos efectos socio-psicológicos en la región. La duración prevista para esta actividad era de 30 minutos y finalmente se requirió el doble del tiempo calculado. Los alumnos realizaron satisfactoriamente la primera parte donde habían tomado sus notas (el $80 \%$ identificó los aspectos claves en la construcción de la narrativa histórica y cinematográfica). La última hora se dedicó a tratar la Constitución de 1889, suprimiendo la parte de trabajo sobre los textos literarios por falta de tiempo. Además de los errores en la estimación inicial del tiempo requerido por el alumnado para completar satisfactoriamente las actividades propuestas, a partir de la tercera sesión detecté que el nivel de concentración de los alumnos se redujo considerablemente. El cansancio acumulado a última hora del turno de mañana, una de las sesiones semanales tenía lugar de 13:00 h. a 15:00 h., y la pérdida del factor novedad afectarían negativamente al rendimiento en los módulos trabajados durante la segunda semana de aplicación del CIMA.

Ciclos de Mejora en el Aula (2020). Experiencias de Innovación Docente de la US Esta obra se distribuye con la licencia Creative Commons 
Por todo ello, en el apartado de valoración de mi experiencia de aplicación del ciclo y de las enseñanzas extraídas para futuros CIMA, en primer lugar, apostaría por reducir los contenidos a trabajar. Esto me llevaría a abreviar el número de problemas planteados, eliminaría el PG y trabajaría sólo con dos problemas (P1 y P2). De ese modo, simplificaría el flujo de retroalimentación circular entre el PG y los P1-P2, usando un planteamiento lineal con los P1P2 y trabajaría el PG durante la extracción de conclusiones. En segundo lugar, observé la necesidad de trabajar expresamente con mis alumnos la "desprogramación" de sus ideas y prejuicios férreamente interiorizados, basados en la creencia de que el aprendizaje histórico se reduce al esquema transmisivo. El docente explica y ellos -asumiendo un papel pasivo- toman notas poniendo el foco sobre los datos más que en los conceptos y procedimientos intelectuales. También hay que incorporar como factor condicionante, en este mismo apartado, que la asignatura pertenece a un Grado que no se orienta específicamente a la formación histórica y la mayoría de los alumnos no se sienten a nivel personal interpelados por el interés en la materia. Por último, dentro de la evaluación, el análisis de los cuestionarios finales demostró no sólo la deseabilidad sino también la viabilidad del modelo basado en la reelaboración de las ideas previas de los estudiantes. Tras trabajar en la categorización de las respuestas de los alumnos por niveles y analizar los obstáculos de aprendizaje (mediante escaleras de aprendizaje), la progresión quedó patente en los porcentajes de alumnos incluidos en los distintos peldaños de las escaleras. Resultados similares se observan en la tabla de progresión individual de los 27 cuestionarios analizados.

Ciclos de Mejora en el Aula (2020). Experiencias de Innovación Docente de la US Esta obra se distribuye con la licencia Creative Commons 

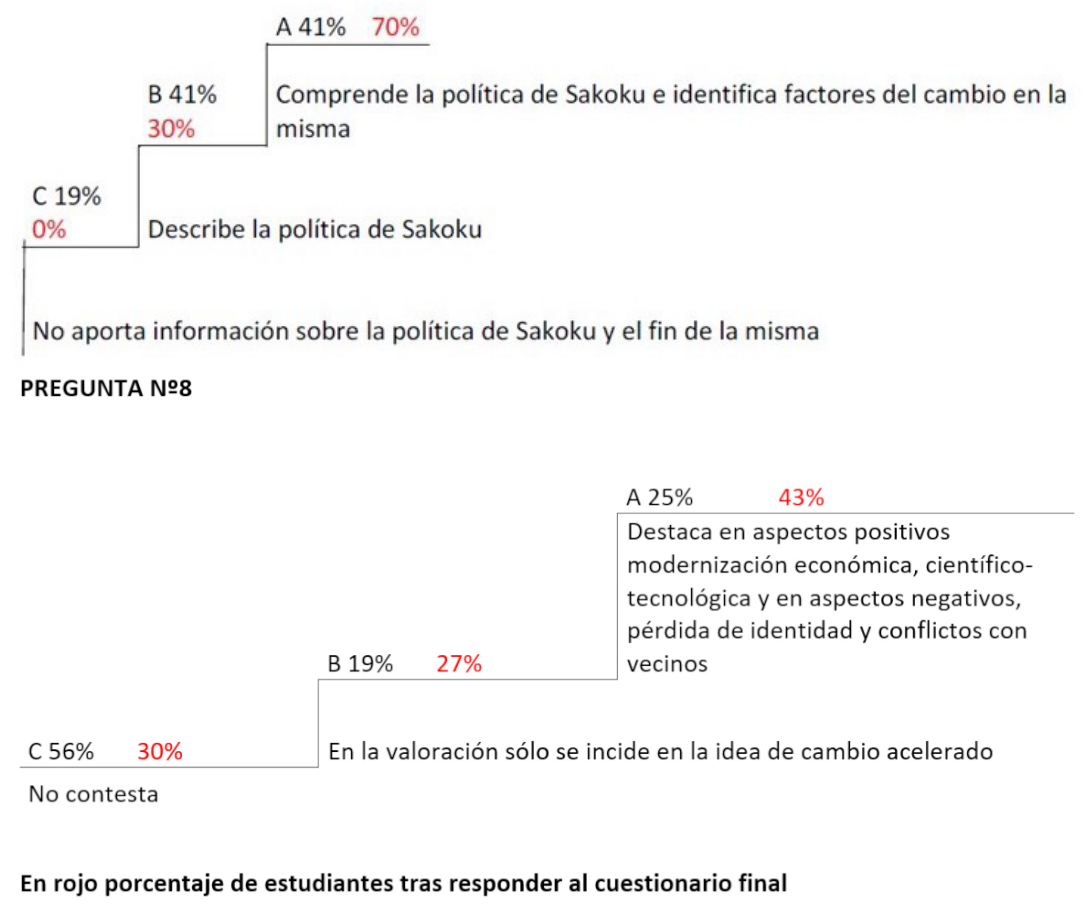

Figura 5. escaleras de aprendizaje

Si tomamos como ejemplo lo sucedido con la progresión reflejada en la primera y última pregunta del cuestionario, más simple y compleja respectivamente, observamos el salto experimentado en cuanto a un modelo de conocimiento descriptivo a otro centrado en el razonamiento, establecimiento de relaciones causales y apuntando a una comprensión profunda de los procesos. En la pregunta número 8 conectada con el PG, se observa cómo se ha incrementado en 18 puntos porcentuales el número de alumnos capaces de alcanzar un nivel de argumentación compleja de sus respuestas a partir de los datos y conceptos históricos trabajados. Además, la mayoría de los alumnos superó el escalón de conocimiento descriptivo de los hechos, empleando un prisma analítico a la hora de expresar lo que habían aprendido.

Ciclos de Mejora en el Aula (2020). Experiencias de Innovación Docente de la US Esta obra se distribuye con la licencia Creative Commons 


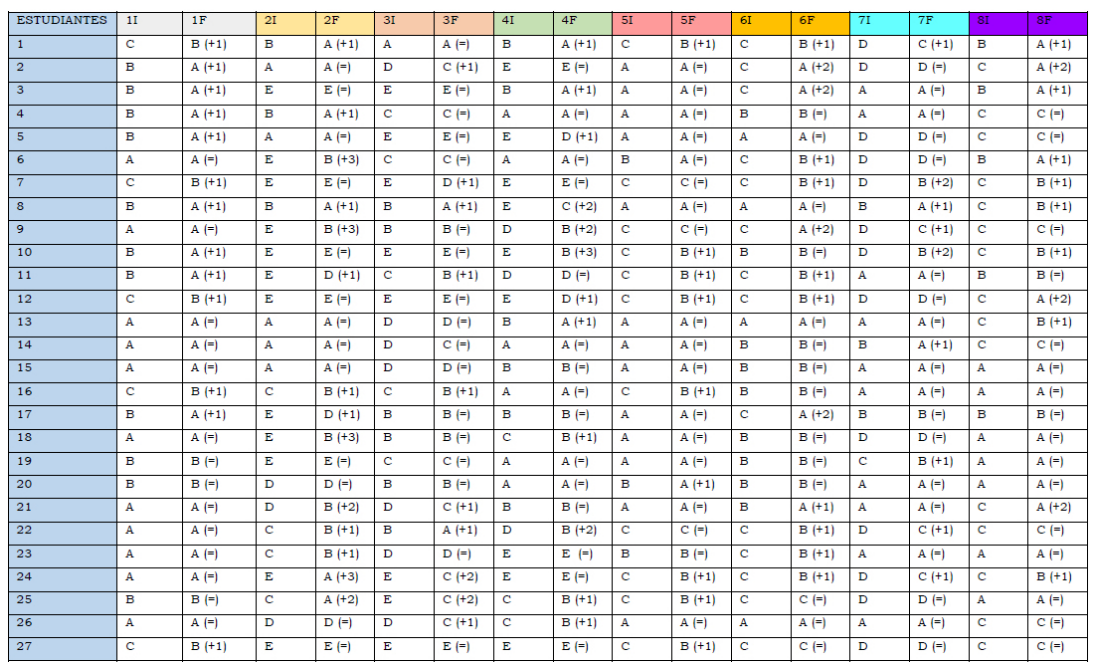

Figura 6. Tabla de progresión individual

\section{Evaluación del CIMA}

En este último apartado de evaluación de la experiencia en su conjunto destacaría como uno de los aspectos claves para la docencia universitaria la exigencia de no descuidar la naturaleza necesariamente bidireccional del proceso de enseñanza-aprendizaje, por muy bien preparados que estén los contenidos y excelentes que sean los enfoques teórico-metodológicos desde los que se aborda la materia. Éstos carecen de sentido cuando no se da el aprendizaje de los alumnos. De ahí la importancia de orientar la selección y organización de los contenidos, el diseño metodológico y las actividades hacia la construcción del conocimiento por parte del estudiante. El método de enseñanza debe fomentar un aprendizaje interactivo y colaborativo entre iguales. El profesor tiene la obligación de velar y asistir para que las condiciones de este se experimenten dentro del aula (Bain, 2005). Una concepción del papel del docente que no implica en absoluto una merma en su importancia dentro del proceso y, que todo lo contario, exige mucho más tiempo y dedicación en la preparación

Ciclos de Mejora en el Aula (2020). Experiencias de Innovación Docente de la US Esta obra se distribuye con la licencia Creative Commons 
de las clases que el modelo de enseñanza meramente transmisivo centrado en el/la profesor/a. El modelo propuesto exige una reflexión mucho más profunda y rigurosa sobre los contenidos y su carácter estructurante. La nueva aproximación hacia estos aspectos, como paso previo al diseño de mis clases, me ha ayudado a reforzar mis objetivos como docente y a articular una metodología coherente con aquéllos. El trabajo por problemas y las herramientas aportadas a los alumnos durante el CIMA se orientan a "enseñarles a aprender", una enseñanza valiosa que encarna uno de los hallazgos y aprendizajes profesionales más relevantes extraídos durante el CIMA y que incorporo como principio didáctico personal. En estas semanas me he familiarizado con nuevos enfoques sobre mi propia práctica docente y adquirido herramientas indispensables para canalizar y tener acceso a esa forma de enseñar. El docente debe partir de las ideas previas del alumnado para, desde ellas, ayudarles a construir un aprendizaje duradero y útil conectado con sus experiencias y horizontes de desarrollo personal (no solamente operante en el ámbito académico). De aquí se extrae otro nuevo principio didáctico personal: el uso de los cuestionarios iniciales y finales en mi práctica docente. Finalmente, como resultado de esta experiencia destacaría el papel y la propia concepción de la evaluación del aprendizaje del alumnado, de su complejidad y riqueza más allá de la calificación sobre los datos retenidos memorísticamente para un momento puntal, coincidente con la realización del examen final. La "evaluación debe ser "para" el aprendizaje y no solo "del" aprendizaje" (Porlán, 2017, p.73).

Palabras Claves: Historia Contemporánea de Asia Oriental, Política, Identidad y Conflicto en Japón siglo XIX, Grado de Estudios de Asia Oriental, Docencia Universitaria, Experimentación docente universitaria

Keywords: Modern History of East Asia, Politics, Identity and Conflict in Japan XIXth Century, East Asian Studies, University Education, Innovation in University Education

Ciclos de Mejora en el Aula (2020). Experiencias de Innovación Docente de la US Esta obra se distribuye con la licencia Creative Commons 


\section{Referencias Bibliográficas}

Bain, K. (2005). Lo que hacen los mejores profesores universitarios. Valencia: Publicaciones Universidad de Valencia.

Finkel, D. (2008). Dar clases con la boca cerrada. Valencia: Publicaciones Universidad de Valencia.

García Martínez, J. (2007). Una estrategia para la enseñanża basada en competencias. X Congreso de Metodología de las Ciencias Sociales y de la Salud. Asociación Española de Metodología de las Ciencias del Comportamiento y Universitat de Barcelona.

García Martínez, J. (2007). Las competencias como foco de organización del Espacio Europeo de Educación Superior. Perficit, 27 (1), 93-114.

Grupo de alto nivel para la modernización de la enseñanza superior de la UE (2013). Enseñar a los profesores a enseñar. Recomendaciones para la mejora de la calidad de la enseñanza y el aprendizaje en la enseñanza superior. Comisión Europea.

Porlán, R. (1993). Constructivismo y escuela. Hacia un modelo de enseñanza-aprendizaje basado en la investigación. Sevilla: Díada.

Porlán, R. Coord. (2017). Enseñanza universitaria. Cómo mejorarla. Madrid: Morata.

Porlán, R. y Martín, J. (1991): El diario del profesor. Un recurso para la investigación en el aula. Sevilla: Díada.

Ciclos de Mejora en el Aula (2020). Experiencias de Innovación Docente de la US Esta obra se distribuye con la licencia Creative Commons 